\title{
Chylothorax Secondary to Spontaneous Rupture of the Cisterna Chyli Treated with Lymphangiography
}

\section{David Zhang1, Kohei Hashimoto ${ }^{1,2}$, Dheeraj K. Rajan ${ }^{3}$ and Michael Augustine Ko ${ }^{1,2^{\star}}$}

${ }^{1}$ Department of Surgery, University of Toronto, Toronto, ON, Canada

${ }^{2}$ Division of Thoracic Surgery, St. Joseph's Health Centre, Toronto, ON, Canada

${ }^{3}$ Division of Vascular \& Interventional Radiology, Department of Medical Imaging, University of Toronto, Toronto, ON, Canada

\begin{abstract}
We report a case of idiopathic chylothorax refractory to surgical management, where the leak was due to a spontaneously ruptured cisterna chyli. Surgical management was unsuccessful, including a left-sided pleuroscopy/ pleurodesis and a right-sided thoracic duct ligation. After a prolonged period of hospitalization, lymphangiography ultimately demonstrated the site of the chyle leak as a spontaneously disrupted cisterna chyli. Due to the degree of disruption, it was not amenable to cannulation and embolization. However, lipiodol injection was sufficient in itself to lead to resolution of the chylothorax. Lymphangiography is a useful modality that can be potentially therapeutic in persistent chylothorax.
\end{abstract}

Keywords: Thoracic surgery; Interventional radiology; Pleural effusion; Chylothorax; Pleuroscopy; Lymphangiography

\section{Case Presentation}

A 44-year-old Asian woman presented to her family physician with a week history of dry cough and dyspnea on exertion. No precipitating factor was known. Her past medical history was significant for an episode of pulmonary tuberculosis treated 6 years prior. A chest X-ray (CXR) demonstrated the presence of a large left pleural effusion, from which biochemical analysis from a thoracentesis confirmed the diagnosis of a chylothorax (Figure 1). Cultures of the pleural fluid were negative, and a CT scan of her chest, abdomen and pelvis revealed no evidence of malignancy. A diagnosis of idiopathic chylothorax was made, and the patient was admitted for non-surgical management via a trial of nihil per os (NPO) and total parenteral nutrition (TPN).

After a week of unsuccessful non-surgical management, it was decided to bring the patient to the operating room, where she underwent a video-assisted left pleuroscopy, decortication, pleurectomy and talc pleurodesis. Intra-operatively a nasogastric tube was inserted, and after instillation of $200 \mathrm{~mL}$ of cream, no leakage of chyle could be seen. Post-operatively, she was started on a trial of oral intake, however, in the following days she developed a recurrent chylous left pleural effusion. She thus restarted a regimen of NPO and TPN, and soon thereafter was brought back to the operating room for a video assisted right-sided thoracic duct ligation.

Although she did well initially, she again failed a second trial of oral intake. After consultation with interventional radiology, she underwent a lymphangiogram with attempted lymphatic embolization. Needle cannulation of the inguinal lymph nodes and injection of $8 \mathrm{cc}$ of lipiodol demonstrated the cisterna chyli to be poorly marginated and diffusely disrupted (Figure 2a). Extravasation of contrast (lipiodol) was found to the left of the disrupted cisterna chyli adjacent to the T11 vertebral body. This was tracking up the retroperitoneum behind the diaphragmatic crus into the left pleural space. Percutaneous fluoroscopic guided attempted cannulation of the cisterna chyli was unsuccessful. After cannulation of the origin of the thoracic duct, injection of contrast demonstrated a successful ligation of the thoracic duct. Ongoing leakage of chyle into the left chest was therefore through these alternate retroperitoneal pathways identified during the lymphangiogram. Embolization therefore could not be performed.

Following the lymphangiogram, however, the chyle leak started to

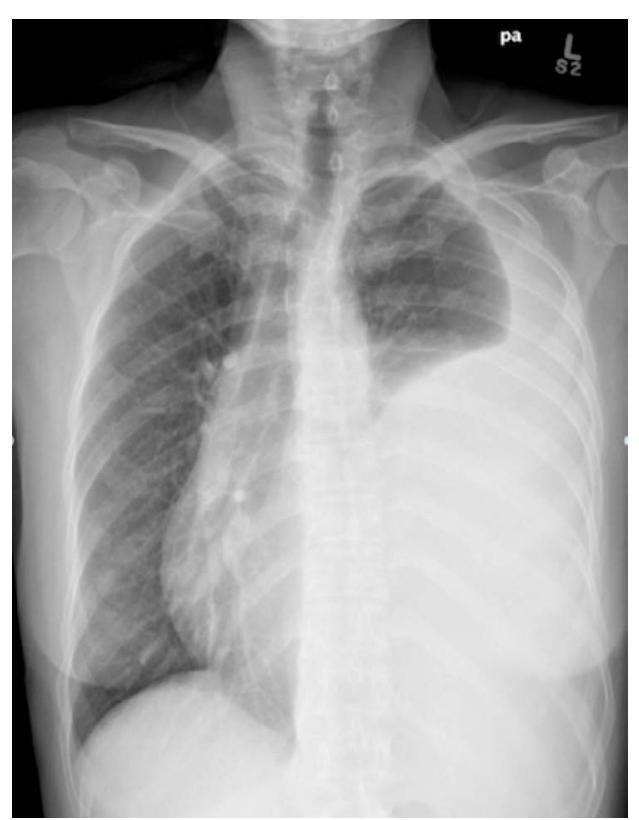

Figure 1: Chest x-ray at presentation demonstrating a large left pleural effusion causing mediastinal shift.

diminish considerably in the week following the procedure. A third trial of diet was started one-week post lymphangiogram, and no further recurrence of chylothorax was observed during the remainder of her hospitalization. Her chest tubes were then removed, and she was discharged home in a stable condition after an 8-week hospitalization.

*Corresponding author: Michael Augustine Ko, Division of Thoracic Surgery, St Joseph's Health Care, Sunnyside Wing, Suite 240, 30 The Queensway, Toronto, ON Canada, Tel: (416) 530-6653; Fax: (416) 530-6114; E-mail: komich@stjoe.on.ca

Received: May 19, 2016; Accepted: June 16, 2016; Published: June 20, 2016

Citation: Zhang D, Hashimoto K, Rajan DK, Ko MA (2016) Chylothorax Secondary to Spontaneous Rupture of the Cisterna Chyli Treated with Lymphangiography. J Pulm Respir Med 6: 355. doi:10.4172/2161-105X.1000355

Copyright: ( $) 2016$ Zhang D, et al. This is an open-access article distributed under the terms of the Creative Commons Attribution License, which permits unrestricted use, distribution, and reproduction in any medium, provided the original author and source are credited. 


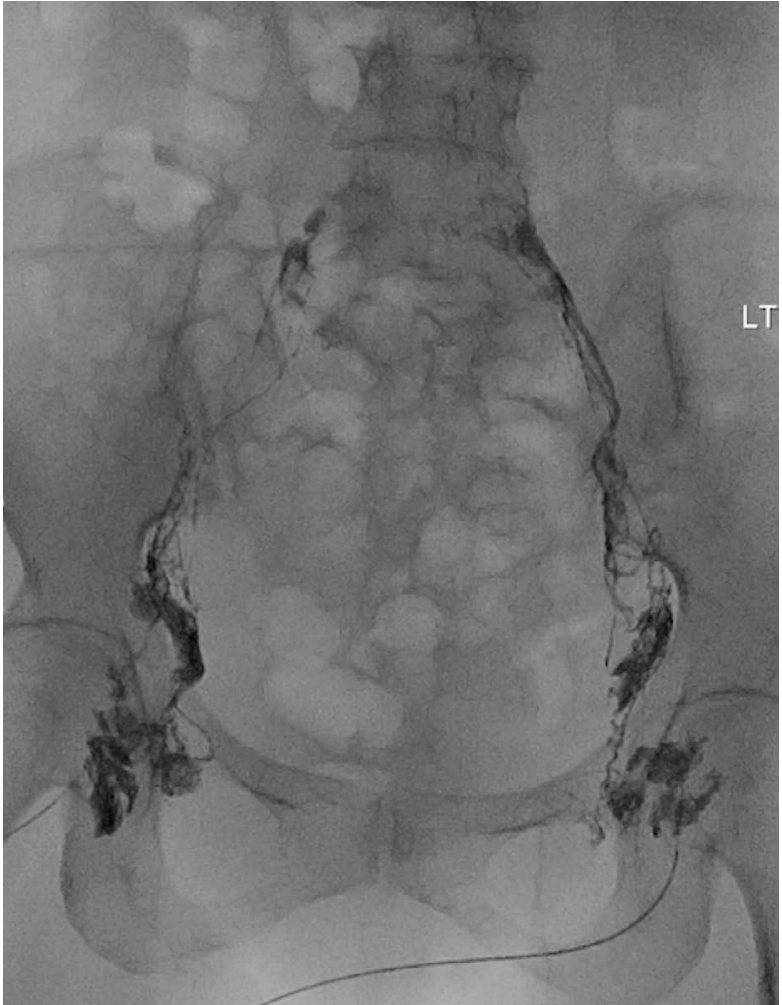

Figure 2a: Lipiodol injection from inguinal lymph nodes via 30 -gauge needle.

Follow-up chest $\mathrm{x}$-ray performed at one month and six months' post admission demonstrated no evidence of recurrent chylothorax.

\section{Discussion}

Chylothorax, the presence of chyle in the pleural space usually develops in the post-operative setting or with malignancy. However, approximately $5 \%$ to $10 \%$ of cases are idiopathic in origin [1]. Spontaneous idiopathic chylothorax is rare in adults, and most reported cases in the literature are successfully treated with thoracic duct ligation [2]. In our patient, two surgical attempts at controlling the chyle leak were unsuccessful, which was ultimately identified on lymphangiogram to be at the level of the cisterna chyli. This had the appearance of being spontaneously ruptured (Figure $2 b$ ). The exact cause for the rupture of the cisterna chyli remains unclear. Perhaps a congenital malformation of the lymphatic system, or perhaps damage to the lymphatic system from prior tuberculosis, may have predisposed to spontaneous disruption. However, the latter hypothesis seems unlikely given that most cases of chylothorax secondary to tuberculosis involve either obstruction of the lymphatic tree or an active tuberculosis infection (as demonstrated by positive cultures or the presence of mycobacterial deoxyribonucleic acid in the pleural fluid [3]). Interestingly, our patient developed chylothorax secondary to retroperitoneal tributaries of the cisterna chyli, rather than through the main thoracic duct per se. This would explain the failure of a successfully performed right-sided thoracic duct ligation to control her chylous effusion. We hypothesize that the negative pressure generated by inspiration allowed the cisterna-pleural fistula to form versus tracking of the chyle from the enclosed retroperitoneal high pressure space to the low pressure pleural space. At the time of writing, this is the first described case of spontaneous cisterna chyli disruption treated with lymphangiography.

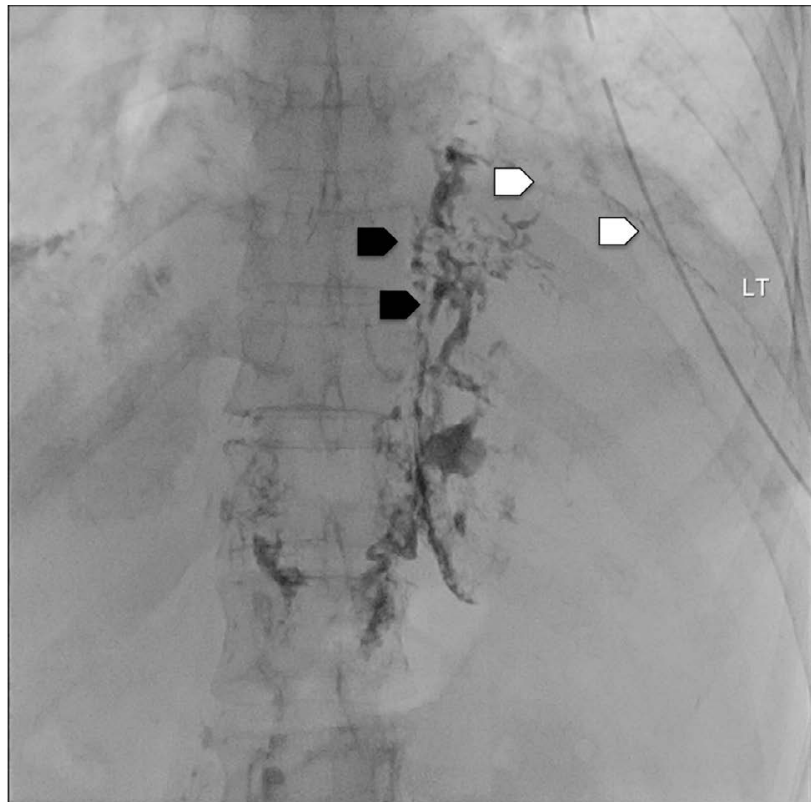

Figure 2b: Extravasation of lipiodol from a diffusely disrupted cisterna chyli (black arrowheads) to the left pleural space through the left diaphragm (white arrowheads).

Thoracic duct embolization has become a reasonable option in the treatment armamentarium for chylothorax in the recent years. When successfully cannulated, embolization of the thoracic duct can lead to resolution of chyle leak in up to $90 \%$ of patients [4]. Interestingly, despite the unsuccessful cannulation of the cisterna chyli in our patient, the injection of lipiodol via inguinal lymph nodes was sufficient to lead to a cessation of chyle leak, a known effect explained by the propensity of lipiodol to form an inflammatory reaction in the soft tissue at the site of leak, which then obstructs the leaking lymphatic vessel [5].

In summary, spontaneous idiopathic chylothorax is rare among adults. When non-operative management fails, perhaps lymphangiography, and possibly thoracic duct embolization, is warranted prior to any surgical intervention in order to identify the precise location of the chyle leak and for the definitive treatment.

\section{References}

1. Doerr CH, Allen MS, Nichols FC 3rd, Ryu JH (2005) Etiology of chylothorax in 203 patients. Mayo Clin Proc 80: 867-870.

2. Desai N, Chaddha U, Desai S, Gable B (2012) Idiopathic chylothorax in a young man. BMJ Case Rep 2012: bcr2012007318.

3. Karapolat S, Sanli A, Onen A (2008) Chylothorax due to tuberculosis lymphadenopathy: report of a case. Surg Today 38: 938-941.

4. Itkin M, Kucharczuk JC, Kwak A, Trerotola SO, Kaiser LR (2010) Nonoperative thoracic duct embolization for traumatic thoracic duct leak: experience in 109 patients. J Thorac Cardiovasc Surg 139: 584-589.

5. Matsumoto T, Yamagami T, Kato T, Hirota T, Yoshimatsu R, et al. (2009) The effectiveness of lymphangiography as a treatment method for various chyle leakages. Br J Radiol 82: 286-290. 